\title{
Contamination, Sources, and Environmental Hazards of Groundwater in Bemetara District, Chhattisgarh, Central India
}

\author{
Nohar Singh Dahariya, Ph.D. 1; Ankit Yadav²; Suryakant Chakradhari³; \\ Khageshwar Singh Patel ${ }^{4}$; Jose Nicolás ${ }^{5}$; Eduardo Yubero; \\ Matini Lautent ${ }^{7}$; and Pablo Martín-Ramos ${ }^{8}$
}

\begin{abstract}
The groundwater of the the Bemetara district of Chhattisgarh in central India over a large area is hard in nature due to its high mineral content. An elevated concentration of $\mathrm{Na}^{+}, \mathrm{Mg}^{2+}, \mathrm{Ca}^{2+}$, and $\mathrm{SO}_{4}^{2-}$ in the groundwater has been observed, falling within the ranges $(n=16)$ 30-437, 43-341, 169-660, and 254-2,330 $\mathrm{mg} \mathrm{L}^{-1}$ with a mean value of $107 \pm 93,117 \pm 69,387 \pm 171$, and 1,059 $\pm 595 \mathrm{mg} \mathrm{L}^{-1}$, respectively. The temporal and spatial variations in the groundwater concentration of species, i.e., $\mathrm{SO}_{4}^{2-}, \mathrm{Cl}^{-}, \mathrm{NO}_{3}^{-}, \mathrm{Na}^{+}, \mathrm{K}^{+}, \mathrm{Mg}^{2+}, \mathrm{Ca}^{2+}, \mathrm{Al}$, and Fe, during the period 2010-2016 are described. The sources of the contaminants and quality of the groundwater are discussed. The environmental hazards of the contaminated water, i.e., land degradation, rusting of buildings and pipes, physiological drought, and ill health of aquatics, birds, and animals, in the Bemetara area are discussed.
\end{abstract}

Author keywords: Groundwater quality; Gypsum; Mineralization; Environmental hazard.

\section{Introduction}

Sulfates occur naturally in numerous minerals, including gypsum $\left(\mathrm{CaSO}_{4} \cdot 2 \mathrm{H}_{2} \mathrm{O}\right)$, epsomite $\left(\mathrm{MgSO}_{4} \cdot 7 \mathrm{H}_{2} \mathrm{O}\right)$, and barite $\left(\mathrm{BaSO}_{4}\right)$, and their dissolved minerals contribute to the mineral content of many drinking waters (Greenwood and Earnshaw 1984). Sulfur is important for humans because it is part of the amino acid methionine, which is an absolute dietary requirement. The permissible limit of $\mathrm{SO}_{4}^{2-}$ in water is $150 \mathrm{mg} \mathrm{L}^{-1}$. Sulfates can contribute to an undesirable taste in water, and intake of sulfate-contaminated drinking water has effects on human health, for example, neurological effects and behavioral changes, disturbance of blood circulation, heart

${ }^{1}$ School of Studies in Chemistry, Pt. Ravishankar Shukla Univ., Raipur, CG 492010, India. Email: nohardahariya@yahoo.in

${ }^{2} \mathrm{Ph}$.D. Student, School of Studies in Environmental Science, Pt. Ravishankar Shukla Univ., Raipur, CG 492010, India. Email: yadavankit .yadav9@gmail.com

${ }^{3} \mathrm{Ph} . \mathrm{D}$. Student, School of Studies in Environmental Science, Pt. Ravishankar Shukla Univ., Raipur, CG 492010, India. Email: suryachakradhari99@gmail.com

${ }^{4}$ Professor Emeritus, School of Studies in Chemistry, Pt. Ravishankar Shukla Univ., Raipur, CG 492010, India (corresponding author). Email: patelkhageshwarsingh@gmail.com

${ }^{5}$ Professor, Dept. of Applied Physics, Miguel Hernandez Univ., Elche, Alicante 03202, Spain. Email: j.nicolas@umh.es

${ }^{6}$ Professor, Dept. of Applied Physics, Miguel Hernandez Univ., Elche, Alicante 03202, Spain. Email: eyubero@umh.es

${ }^{7}$ Professor, Dept. of Exact Sciences, Marien Ngouabi Univ., Brazzaville BP 69, Congo. Email: matinilaurent@yahoo.fr

${ }^{8}$ Professor, Dept. of Agricultural and Environmental Sciences, Escuela Politécnica Superior, Instituto de Investigación en Ciencias Ambientales, Univ. of Zaragoza, Carretera de Cuarte, s/n, Huesca 22071, Spain. ORCID: https://orcid.org/0000-0003-2713-2786. Email: pmr@unizar.es

Note. This manuscript was submitted on February 27, 2019; approved on June 25, 2019; published online on September 9, 2019. Discussion period open until February 9, 2020; separate discussions must be submitted for individual papers. This paper is part of the Journal of Hazardous, Toxic, and Radioactive Waste, (C) ASCE, ISSN 2153-5493. damage, effects on eyes and eyesight, reproductive failure, damage to the immune system, stomach and gastrointestinal disorders, damage to liver and kidney function, hearing defects, disturbance of the hormonal metabolism, dermatological effects, and suffocation and lung embolism (Backer 2008; Burgess et al. 2010). Sulfate solution in contact with concrete can cause chemical changes to the cement, which can lead to significant microstructural effects, resulting in a weakening of the cement binder (Atasoy and Yesilnacar 2010; Lorente et al. 2011; Pradhan 2014). Naturally occurring sulfate-contaminated water has been reported in some regions of the world (Seller and Canter 1980; MDH 2008; Horst et al. 2011; Mubarak et al. 2015; Han et al. 2016; Stanton et al. 2017). Similarly, groundwater containing high concentrations of $\mathrm{Cl}^{-}, \mathrm{Na}, \mathrm{Mg}$, and $\mathrm{Ca}$ in other locations of the world has been observed (Kukillaya et al. 2004; Hajalilou and Khaleghi 2009; van Weert et al. 2009; Yamakanamardi et al. 2011; Razowska-Jaworek 2014; Bhandary et al. 2018).

In the Bemetara district of central India, there is a high incidence of gastrointestinal disorders in humans and livestock, together with serious impacts on wet and bush land ecosystems and a marked corrosion of materials (e.g., houses, pipelines, buildings, roads, water supply systems), all attributable to water pollution. Nonetheless, a detailed investigation of the mineral contamination of water in this region has not been reported to date. In this work, the contamination variations, sources, and toxicity of groundwater from this region of central India are discussed.

\section{Materials and Methods}

\section{Study Area}

The Bemetara district in the Indian state of Chhattisgarh $\left(21.70^{\circ} \mathrm{N} 81.53^{\circ} \mathrm{E}\right)$ was selected for the proposed investigation owing to the high salt content in the water (Fig. 1). The district consists of four blocks, Bemetara, Nawagarh, Saja, and Berla. The area is occupied by mesoproterozoic sedimentary hard rocks over approximately $2.8 \times 10^{3} \mathrm{~km}^{2}$, with a population of around 1 million 


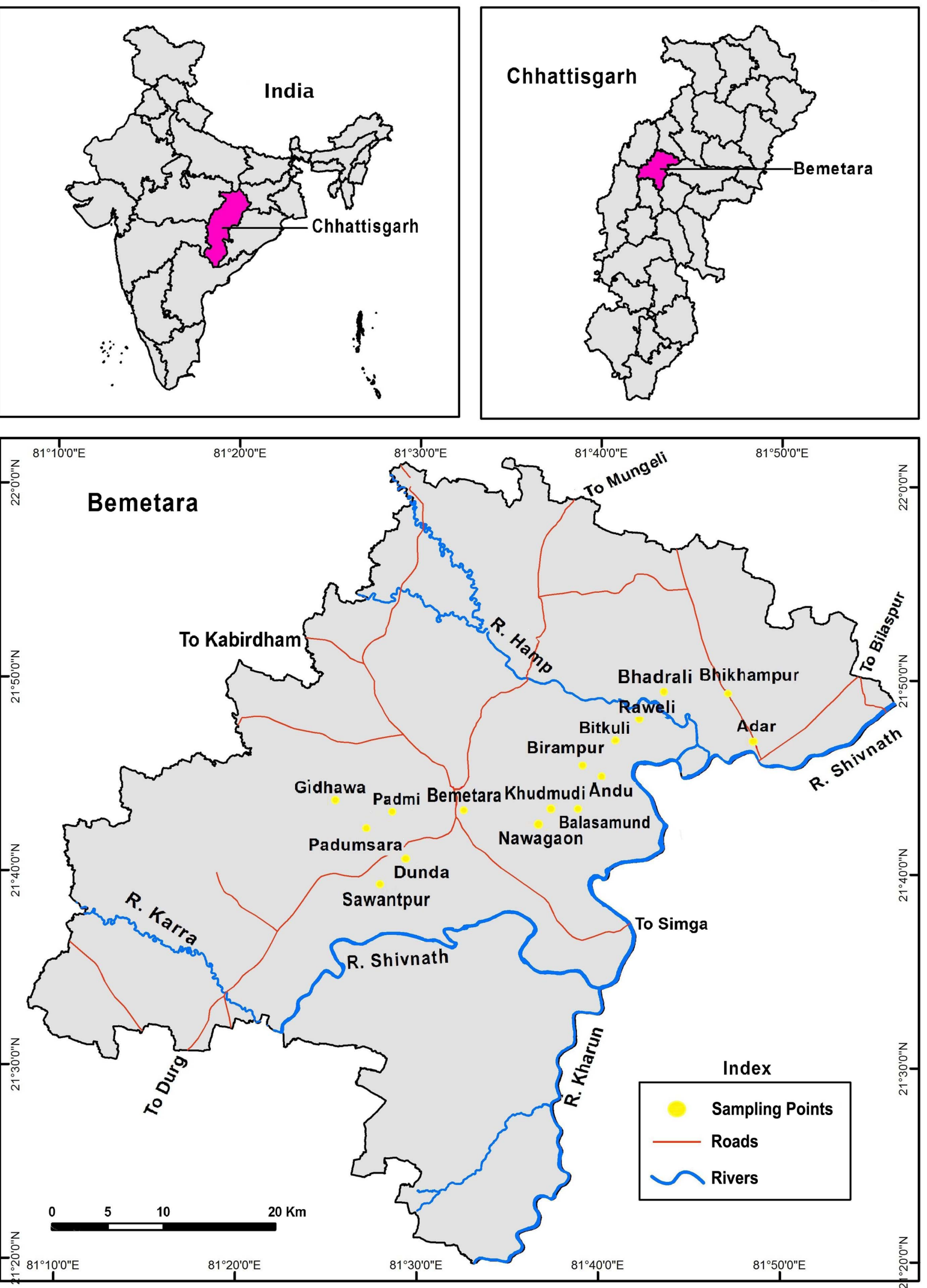

Fig. 1. Sampling locations in Bemetara area, Durg, Chhattisgarh, India. 
inhabitants. The water quality, plant biodiversity, and food grain yields in this area have been negatively affected due to the precipitation of minerals in the water.

\section{Sample Collection}

The sampling network for water collection is shown in Fig. 1. Groundwater samples were collected in duplicate from 16 locations (cities and villages) using the method of Nielsen and Nielsen (2006). Polyethylene bottles were used for water collection, after rinsing three times with the water to be sampled prior to collection. Samples sent to the laboratory were stored at $-4^{\circ} \mathrm{C}$. For seasonal variation studies, water was collected in three seasons: monsoon (September), postmonsoon (January), and premonsoon (May) in 2009-2010. For the temporal variation investigation, water was collected in the postmonsoon season (January) from 2011 to 2016. Physical parameters [viz., $\mathrm{pH}$, temperature (T), electrical conductivity (EC), dissolved oxygen (DO), and oxidation-reduction potential $(\mathrm{ORP})]$ were measured on the spot immediately after water sampling.

\section{Analyses}

Total dissolved solids (TDS) were determined by evaporation of the water samples, previously filtered through a glass fiber filter, and by drying until a constant weight was achieved (APHA 2005). Total hardness (TH) and total alkalinity (TA) were determined using titration methods (Nollet Leo and De Gelder Leen 2007). Ions (viz., $\mathrm{Cl}^{-}, \mathrm{NO}_{3}^{-}, \mathrm{SO}_{4}^{2-}, \mathrm{Na}^{+}, \mathrm{K}^{+}, \mathrm{Mg}^{2+}$, and $\mathrm{Ca}^{2+}$ ) were analyzed by ion chromatography using a Dionex DX1100 (Sunnyvale, California) apparatus equipped with anion and cation separation columns and a conductivity detector. Metals, i.e., Al and Fe, were analyzed with a GBC atomic absorption spectrometer (AAS).

\section{Water Indices}

To assess water quality, various indices, viz., sodium adsorption ratio (SAR), sodium hazard $(\mathrm{SH})$, magnesium hazard $(\mathrm{MH})$, permeability index (PI), Kelly's ratio (KR), and water quality index (WQI), were calculated, according to the following equations:

$$
\begin{gathered}
\mathrm{SAR}=\frac{\mathrm{Na}^{+}}{\sqrt{\frac{\mathrm{Ca}^{2+}+\mathrm{Mg}^{2+}}{2}}} \\
\mathrm{SH}=\frac{\mathrm{Na}^{+}+\mathrm{K}^{+}}{\mathrm{Na}^{+}+\mathrm{K}^{+}+\mathrm{Mg}^{2+}+\mathrm{Ca}^{2+}} \cdot 100 \\
\mathrm{MH}=\frac{\mathrm{Mg}^{2+}}{\mathrm{Ca}^{2+}+\mathrm{Mg}^{2+}} \cdot 100 \\
\mathrm{PI}=\frac{\mathrm{Na}^{+}+\sqrt{\mathrm{HCO}_{3}^{-}}}{\mathrm{Ca}^{2+}+\mathrm{Mg}^{2+}+\mathrm{Na}^{+}} \cdot 100 \\
\mathrm{KR}=\frac{\mathrm{Na}^{+}}{\mathrm{Mg}^{2+}+\mathrm{Ca}^{2+}}
\end{gathered}
$$

where concentrations are expressed in meq $\mathrm{L}^{-1}$.

The WQI was evaluated using a weighed arithmetic method with 10 parameters ( $\mathrm{pH}, \mathrm{DO}, \mathrm{EC}$, hardness, alkalinity, $\mathrm{Mg}^{2+}, \mathrm{Ca}^{2+}$, $\mathrm{Cl}^{-}, \mathrm{NO}_{3}^{-}$, and $\mathrm{SO}_{4}^{2-}$ ), using the following expression (BIS 2009; WHO 2011):

$$
\mathrm{WQI}=\frac{\sum \mathrm{q}_{\mathrm{n}} \cdot \mathrm{W}_{\mathrm{n}}}{\sum \mathrm{W}_{\mathrm{n}}}
$$

where $q_{\mathrm{n}}=\left(V_{n}-V_{i o}\right) /\left(S_{n}-V_{i o}\right) \cdot 100$ is the quality rating of the $n$th parameter; and $V_{n}, S_{n}, V_{i o}$, and $W_{n}$ are the estimated value, standard permissible value, ideal value of pure water, and unit weight of $n$th parameter, respectively. $V_{i o}$ is 0 for all parameters, except for $\mathrm{pH}$ and dissolved oxygen (in which it is 7.0 and $14.6 \mathrm{mg} \mathrm{L}^{-1}$, respectively).

\section{Statistical Analysis}

Aqachem software (Waterloo Hydrogeologic) was used for the preparation of piper diagrams. Multivariate statistical modelsfactor analysis (FA) and hierarchical cluster analysis (HCA) —were used for the source apportionment of ions and metals, in agreement with Shrestha and Kazama (2007) and Hajalilou and Khaleghi (2009), using STATISTICA version 7.1 software (Dell, Statsoft).

\section{Results and Discussion}

\section{Characteristics of Water}

The physical characteristics of the tube wells and groundwater in the postmonsoon period, January 2010, are presented in Table 1. The newest was 10 years old, while the oldest was 20. Depths ranged from 46 to $91 \mathrm{~m}$. A slight variation in temperature (T) was observed, with values ranging from $24.1^{\circ} \mathrm{C}$ to $28.1^{\circ} \mathrm{C}$. The $\mathrm{pH}$ values of the groundwater samples were in the 7.3-7.8 range, with a mean value of $7.6 \pm 0.1$, so all groundwater samples were within the prescribed desirable range (6.5-9.2) for drinking water (BIS 2009; WHO 2011).

Very high values of EC and TDS were observed and ranged from 892 to $3,930 \mu \mathrm{S} \mathrm{cm}^{-1}$ and from 1,205 to $5,362 \mathrm{mg} \mathrm{L}^{-1}$, respectively. The highest EC and TDS values corresponded to a tube well in Location 12, the closest (approximately $20 \mathrm{~km}$ ) to Shivnath River. The EC value was well correlated ( $r=0.98)$ with the TDS of the water. The mean EC and TDS values $\left(1,997 \pm 405 \mu \mathrm{S} \mathrm{cm}^{-1}\right.$ and $2,677 \pm 582 \mathrm{mg} \mathrm{L}^{-1}$, respectively) in the groundwater samples were well above the allowed limits of $300 \mu \mathrm{S} \mathrm{cm}^{-1}$ and $500 \mathrm{mg} \mathrm{L}^{-1}$, respectively (BIS 2009; WHO 2011). According to the salinity classification by Davis and De Wiest (1966), the water of the area under study would fall under the moderately saline category.

The DO and ORP values ranged from 4.9 to $6.3 \mathrm{mg} \mathrm{L}^{-1}$ and from 135 to $240 \mathrm{mV}$, respectively. The ORP values were at least three times lower than the recommended value of $650 \mathrm{mV}$. The DO and ORP values showed moderate negative correlations with the tube well depth and TDS ( $r=-0.82$ and $r=-0.92$, respectively).

Total alkalinity (TA) is mainly derived from the dissolution of carbonate minerals and from $\mathrm{CO}_{2}$ present in the atmosphere and in soil above the water table. The TA values for the groundwater samples ranged from 425 to $1,417 \mathrm{mg} \mathrm{L}^{-1}$ as $\mathrm{CaCO}_{3}$. The highest TA value, observed at Location 4, may be due to cattle waste contamination with carbonate and bicarbonate ions. All TA values were found to be several-fold higher than the recommended value (120 mg L ${ }^{-1}$ ) (BIS 2009; WHO 2011).

Total hardness (TH) index values, which relate to $\mathrm{Mg}^{2+}$ and $\mathrm{Ca}^{2+}$ concentrations, varied from 393 to $1,500 \mathrm{mg} \mathrm{L}^{-1}$, expressed as $\mathrm{CaCO}_{3}$. The highest $\mathrm{TH}$ value of the water was observed at Location 1, which may be tentatively ascribed to the extraction of large amounts of water for irrigation purposes. TH showed a fair correlation $(r=0.60)$ with TA, perhaps due to buffering of $\mathrm{Mg}^{2+}$ and $\mathrm{Ca}^{2+}$. The water from all tube wells can be regarded as extremely hard (>180 $\mathrm{mg} \mathrm{L}^{-1} \mathrm{CaCO}_{3}$ ), according to Doneen (1964).

The chemical characteristics of the groundwater samples collected in the postmonsoon season are presented in Table 2. 
Table 1. Physical characteristics of tube well and groundwater, January 2010

\begin{tabular}{|c|c|c|c|c|c|c|c|c|c|c|c|}
\hline Serial Number & Location & $\begin{array}{c}\text { Age } \\
\text { (years) }\end{array}$ & $\begin{array}{l}\text { Depth } \\
\text { (m) }\end{array}$ & $\mathrm{T}\left({ }^{\circ} \mathrm{C}\right)$ & $\mathrm{pH}$ & $\begin{array}{c}\mathrm{DO} \\
\left(\mathrm{mg} \mathrm{L}^{-1}\right)\end{array}$ & $\begin{array}{l}\text { ORP } \\
(\mathrm{mV})\end{array}$ & $\begin{array}{c}\mathrm{EC} \\
\left(\mu \mathrm{S} \mathrm{cm}^{-1}\right)\end{array}$ & $\begin{array}{c}\mathrm{TDS} \\
\left(\mathrm{mg} \mathrm{L}^{-1}\right)\end{array}$ & $\begin{array}{c}\mathrm{TA} \\
\left(\mathrm{mg} \mathrm{L}^{-1}\right)\end{array}$ & $\begin{array}{c}\mathrm{TH} \\
\left(\mathrm{mg} \mathrm{L}^{-1}\right)\end{array}$ \\
\hline 1 & Padumsara & 20 & 61 & 25.3 & 7.5 & 5.2 & 139 & 2,990 & 4,100 & 1,413 & 1,500 \\
\hline 2 & Bhadrali & 15 & 61 & 25.4 & 7.5 & 5.3 & 187 & 1,880 & 2,393 & 788 & 833 \\
\hline 3 & Padmi & 15 & 76 & 26.8 & 7.5 & 5.2 & 181 & 2,100 & 2,806 & 935 & 1,000 \\
\hline 4 & Gidhwa & 5 & 91 & 27.8 & 7.7 & 5.0 & 145 & 2,650 & 3,958 & 1,417 & 1,440 \\
\hline 5 & Duda & 20 & 61 & 25.6 & 7.8 & 5.4 & 205 & 1,560 & 1,911 & 717 & 645 \\
\hline 6 & Bhikhanpur & 15 & 61 & 25.8 & 7.6 & 5.8 & 238 & 1,235 & 1,537 & 747 & 448 \\
\hline 7 & Bemetara & 10 & 91 & 28.1 & 7.6 & 5.1 & 198 & 1,580 & 2,423 & 425 & 940 \\
\hline 8 & Nawagaon & 20 & 46 & 24.6 & 7.5 & 6.3 & 215 & 2,100 & 3,223 & 435 & 1,193 \\
\hline 9 & Raweli & 20 & 46 & 24.7 & 7.4 & 5.9 & 238 & 931 & 1,373 & 595 & 470 \\
\hline 10 & Khurmuri & 20 & 46 & 24.6 & 7.6 & 5.8 & 240 & 892 & 1,205 & 795 & 393 \\
\hline 11 & Balsamund & 15 & 61 & 24.7 & 7.6 & 5.3 & 210 & 1,920 & 2,079 & 570 & 830 \\
\hline 12 & Andu & 12 & 61 & 24.6 & 7.7 & 5.9 & 135 & 3,930 & 5,362 & 1,352 & 1,480 \\
\hline 13 & Sawantpur & 15 & 61 & 24.5 & 7.3 & 5.7 & 221 & 1,250 & 1,513 & 760 & 490 \\
\hline 14 & Birampur & 20 & 61 & 24.4 & 7.6 & 5.6 & 154 & 2,780 & 3,813 & 737 & 1,485 \\
\hline 15 & Adar & 20 & 46 & 24.1 & 7.8 & 6.2 & 214 & 1,530 & 1,841 & 657 & 580 \\
\hline \multirow[t]{5}{*}{16} & Bitkuli & 3.0 & 91 & 28.1 & 7.8 & 4.9 & 161 & 2,620 & 3,301 & 1,217 & 1,093 \\
\hline & Minimum & 3.0 & 46 & 24.1 & 7.3 & 4.9 & 135 & 892 & 1,205 & 425 & 293 \\
\hline & Maximum & 20 & 91 & 28.1 & 7.8 & 6.3 & 240 & 3,930 & 5,362 & 1,417 & 1,500 \\
\hline & Mean & 15 & 64 & 25.6 & 7.6 & 5.5 & 193 & 1,997 & 2,677 & 848 & 926 \\
\hline & Standard deviation, \pm & 5 & 16 & 1.4 & 0.1 & 0.4 & 36 & 827 & 1,188 & 329 & 404 \\
\hline
\end{tabular}

Extremely high $\mathrm{SO}_{4}^{2-}$ concentrations were detected, with values ranging from 254 to $2,330 \mathrm{mg} \mathrm{L}^{-1}$, well above the permissible limit of $150 \mathrm{mg} \mathrm{L}^{-1}$ (BIS 2009; WHO 2011). $\mathrm{SO}_{4}^{2-}$ content showed a moderate/high correlation with $\mathrm{Mg}^{2+}$ and $\mathrm{Ca}^{2+}$ contents $(r=0.86$ and $r=0.90$, respectively), suggesting that it may come from the dissolution of gypsum.

Chloride in groundwater may originate from different sources, which include the dissolution of halite and related minerals. Chloride concentrations in the groundwater samples ranged from 7.0 to $76 \mathrm{mg} \mathrm{L}^{-1}$, much lower than the recommended limit of $250 \mathrm{mg} \mathrm{L}^{-1}$ (BIS 2009; WHO 2011).

Sodium concentration varied from 30 to $437 \mathrm{mg} \mathrm{L}^{-1}$. Such an extremely high concentration of $\mathrm{Na}^{+}$(much higher than the
$180 \mathrm{mg} \mathrm{L}^{-1}$ limit for drinking water) was observed in Location 12 , which, as noted earlier, lies closer to the river. It is worth noting that high $\mathrm{Na}^{+}$concentrations (beyond $20 \mathrm{mg} \mathrm{L}^{-1}$ ) in water is associated with various health hazards (e.g., hypertension, heart diseases, kidney problems). The $\mathrm{Na}^{+} / \mathrm{Cl}^{-}$ratios ranged from 1.3 to 48 . Since $\mathrm{Na}^{+}$concentrations exceed $\mathrm{Cl}^{-}$concentrations in all samples under consideration, a geogenic origin may be presumed. Potassium concentrations were very low in the samples under study, in the $2.1-10.4 \mathrm{mg} \mathrm{L}^{-1}$ range. The $\mathrm{Na}^{+} / \mathrm{K}^{+}$ratio ranged from 10 to 190. Magnesium and $\mathrm{Ca}^{2+}$ concentrations ranged from 43 to $341 \mathrm{mg} \mathrm{L}^{-1}$ and from 169 to $660 \mathrm{mg} \mathrm{L}^{-1}$, beyond the permissible limits of 30 and $75 \mathrm{mg} \mathrm{L}^{-1}$, respectively. The highest $\mathrm{Mg}^{2+}$ and $\mathrm{Ca}^{2+}$ concentrations were again observed from the water

Table 2. Chemical characteristics of the groundwater, January $2010-2016\left(\mathrm{mg} \mathrm{L}^{-1}\right)$

\begin{tabular}{|c|c|c|c|c|c|c|c|c|c|c|c|c|}
\hline Year & Serial Number & $\mathrm{Cl}^{-}$ & $\mathrm{NO}_{3}^{-}$ & $\mathrm{SO}_{4}^{2-}$ & $\mathrm{HCO}_{3}^{-}$ & $\mathrm{NH}_{4}^{+}$ & $\mathrm{Na}^{+}$ & $\mathrm{K}^{+}$ & $\mathrm{Mg}^{2+}$ & $\mathrm{Ca}^{2+}$ & $\mathrm{Al}$ & $\mathrm{Fe}$ \\
\hline \multirow[t]{20}{*}{2010} & 1 & 13 & 3.6 & 1,610 & 848 & 1.2 & 133 & 6.7 & 139 & 660 & 1.69 & 0.74 \\
\hline & 2 & 36 & 11.2 & 924 & 473 & 1.9 & 91 & 5.3 & 96 & 354 & 1.38 & 0.45 \\
\hline & 3 & 23 & 4.7 & 1,109 & 561 & 1.8 & 94 & 4.8 & 108 & 430 & 1.57 & 0.32 \\
\hline & 4 & 46 & 5.6 & 1,489 & 850 & 1.4 & 120 & 5.2 & 169 & 610 & 1.66 & 0.34 \\
\hline & 5 & 7.0 & 18.4 & 696 & 430 & 2.6 & 74 & 5.7 & 98 & 259 & 1.28 & 0.43 \\
\hline & 6 & 29 & 4.3 & 427 & 448 & 1.8 & 116 & 6.1 & 64 & 183 & 1.13 & 0.45 \\
\hline & 7 & 48 & 12.2 & 1,153 & 255 & 1.2 & 41 & 3.6 & 96 & 407 & 1.25 & 0.62 \\
\hline & 8 & 65 & 4.7 & 1,621 & 261 & 1.6 & 85 & 3.2 & 129 & 513 & 1.34 & 0.73 \\
\hline & 9 & 40 & 4.4 & 438 & 357 & 1.1 & 48 & 2.5 & 50 & 202 & 1.17 & 0.38 \\
\hline & 10 & 27 & 5.5 & 254 & 477 & 1.8 & 30 & 5.2 & 43 & 169 & 1.09 & 0.37 \\
\hline & 11 & 76 & 17.3 & 736 & 342 & 4.0 & 98 & 3.2 & 111 & 343 & 1.31 & 0.42 \\
\hline & 12 & 14 & 6.8 & 2,330 & 811 & 2.9 & 437 & 4.0 & 341 & 519 & 1.71 & 0.90 \\
\hline & 13 & 73 & 4.9 & 385 & 456 & 2.6 & 66 & 2.5 & 68 & 201 & 1.19 & 0.37 \\
\hline & 14 & 42 & 1.2 & 1,808 & 442 & 2.1 & 89 & 3.5 & 130 & 657 & 1.74 & 0.83 \\
\hline & 15 & 32 & 3.9 & 700 & 394 & 2.4 & 76 & 2.1 & 89 & 233 & 1.27 & 0.42 \\
\hline & 16 & 23 & 4.2 & 1,265 & 730 & 2.9 & 120 & 10.4 & 134 & 459 & 1.61 & 0.54 \\
\hline & Minimum & 7 & 1.2 & 254 & 255 & 1.1 & 30 & 2.1 & 43 & 169 & 1.09 & 0.32 \\
\hline & Maximum & 76 & 18.4 & 2,330 & 850 & 4.0 & 437 & 10.4 & 341 & 660 & 1.74 & 0.9 \\
\hline & Mean & 37 & 7 & 1,059 & 508 & 2.1 & 107 & 4.6 & 117 & 387 & 1.40 & 0.52 \\
\hline & Standard deviation, \pm & 21 & 5 & 595 & 197 & 0.8 & 93 & 2.1 & 69 & 171 & 0.23 & 0.19 \\
\hline
\end{tabular}


Table 2. (Continued.)

\begin{tabular}{|c|c|c|c|c|c|c|c|c|c|c|c|c|}
\hline Year & Serial Number & $\mathrm{Cl}^{-}$ & $\mathrm{NO}_{3}^{-}$ & $\mathrm{SO}_{4}^{2-}$ & $\mathrm{HCO}_{3}^{-}$ & $\mathrm{NH}_{4}^{+}$ & $\mathrm{Na}^{+}$ & $\mathrm{K}^{+}$ & $\mathrm{Mg}^{2+}$ & $\mathrm{Ca}^{2+}$ & $\mathrm{Al}$ & $\mathrm{Fe}$ \\
\hline \multirow[t]{20}{*}{2011} & 1 & 86 & 18 & 2,034 & 510 & 6.4 & 156 & 6.1 & 164 & 718 & 1.42 & 0.46 \\
\hline & 2 & 30 & 14 & 896 & 606 & 3.2 & 42 & 4.4 & 104 & 156 & 1.26 & 0.56 \\
\hline & 3 & 142 & 26 & 1,015 & 334 & 2.5 & 118 & 6.5 & 122 & 520 & 1.40 & 0.78 \\
\hline & 4 & 49 & 7.2 & 1,240 & 512 & 2.4 & 146 & 4.3 & 132 & 252 & 1.34 & 0.36 \\
\hline & 5 & 32 & 1.8 & 1,108 & 488 & 1.9 & 86 & 12 & 128 & 274 & 1.47 & 0.62 \\
\hline & 6 & 28 & 9.2 & 450 & 448 & 6.8 & 68 & 5.6 & 24 & 267 & 1.34 & 0.56 \\
\hline & 7 & 108 & 22 & 852 & 488 & 1.4 & 44 & 8.4 & 65 & 180 & 1.42 & 0.72 \\
\hline & 8 & 70 & 3.2 & 450 & 856 & 2.8 & 112 & 6.2 & 162 & 316 & 1.34 & 0.68 \\
\hline & 9 & 38 & 2.6 & 315 & 642 & 4.2 & 64 & 6.7 & 46 & 88 & 1.44 & 0.76 \\
\hline & 10 & 74 & 12 & 1,835 & 340 & 5.6 & 78 & 15 & 86 & 622 & 1.40 & 0.52 \\
\hline & 11 & 88 & 0.94 & 625 & 376 & 2.4 & 72 & 7.8 & 142 & 412 & 1.46 & 0.68 \\
\hline & 12 & 46 & 0.85 & 2,940 & 590 & 9.2 & 522 & 7.4 & 412 & 714 & 1.55 & 0.52 \\
\hline & 13 & 76 & 18 & 556 & 620 & 3.3 & 70 & 12 & 96 & 146 & 1.38 & 0.64 \\
\hline & 14 & 52 & 0.54 & 1,912 & 302 & 7.4 & 126 & 13 & 158 & 716 & 1.45 & 0.68 \\
\hline & 15 & 60 & 0.78 & 1,735 & 352 & 6.5 & 109 & 10 & 134 & 508 & 1.35 & 0.66 \\
\hline & 16 & 38 & 4.7 & 1,320 & 460 & 2.4 & 132 & 5.5 & 156 & 252 & 1.36 & 0.42 \\
\hline & Minimum & 28 & 0.5 & 315 & 302.0 & 1.4 & 42 & 4.3 & 24 & 88 & 1.30 & 0.36 \\
\hline & Maximum & 142 & 26.0 & 2,940 & 856.0 & 9.2 & 522 & 15.0 & 412 & 718 & 1.60 & 0.78 \\
\hline & Mean & 64 & 8.9 & 1,205 & 495.3 & 4.3 & 122 & 8.2 & 133 & 384 & 1.40 & 0.60 \\
\hline & Standard deviation, \pm & 32 & 8.5 & 723 & 144.9 & 2.4 & 112 & 3.3 & 85 & 220 & 0.10 & 0.12 \\
\hline \multirow[t]{20}{*}{2012} & 1 & 98 & 22 & 2,802 & 578 & 5.6 & 112 & 7.2 & 110 & 918 & 1.24 & 0.42 \\
\hline & 2 & 28 & 12 & 1,044 & 642 & 4.2 & 82 & 5.1 & 120 & 170 & 1.58 & 0.39 \\
\hline & 3 & 158 & 37 & 708 & 320 & 3.6 & 144 & 5.4 & 96 & 234 & 1.66 & 1.04 \\
\hline & 4 & 58 & 6.20 & 1,516 & 576 & 3.8 & 162 & 3.2 & 180 & 345 & 1.46 & 0.41 \\
\hline & 5 & 42 & 2.12 & 1,256 & 552 & 2.2 & 92 & 10 & 152 & 280 & 1.88 & 0.72 \\
\hline & 6 & 38 & 12.12 & 430 & 488 & 4.6 & 98 & 3.6 & 42 & 340 & 1.18 & 0.32 \\
\hline & 7 & 118 & 24.4 & 512 & 476 & 2.1 & 22 & 5.1 & 120 & 204 & 2.02 & 1.02 \\
\hline & 8 & 76 & 4.8 & 466 & 930 & 4.5 & 52 & 5.2 & 44 & 208 & 1.26 & 0.72 \\
\hline & 9 & 36 & 3.2 & 244 & 714 & 2.3 & 56 & 3.4 & 76 & 108 & 2.14 & 0.88 \\
\hline & 10 & 90 & 10.50 & 2,352 & 328 & 4.4 & 58 & 12 & 115 & 814 & 1.12 & 0.64 \\
\hline & 11 & 64 & 1.12 & 1,030 & 422 & 2.7 & 130 & 5.1 & 48 & 525 & 1.46 & 0.43 \\
\hline & 12 & 60 & 1.24 & 3,918 & 624 & 8.8 & 224 & 6.2 & 332 & 892 & 2.05 & 0.42 \\
\hline & 13 & 85 & 25 & 690 & 772 & 4.6 & 94 & 8.2 & 116 & 180 & 1.48 & 0.67 \\
\hline & 14 & 58 & 0.54 & 2,260 & 262 & 6.7 & 90 & 10 & 245 & 996 & 2.04 & 0.81 \\
\hline & 15 & 52 & 1.12 & 1,980 & 334 & 5.4 & 102 & 7.3 & 167 & 602 & 0.96 & 0.66 \\
\hline & 16 & 42 & 5.02 & 1,378 & 504 & 1.9 & 124 & 3.2 & 188 & 260 & 1.60 & 0.38 \\
\hline & Minimum & 28 & 0.54 & 244 & 262 & 1.9 & 22 & 3.2 & 42 & 108 & 0.96 & 0.32 \\
\hline & Maximum & 158 & 37 & 3,918 & 930 & 8.8 & 224 & 12.4 & 332 & 996 & 2.14 & 1.04 \\
\hline & Mean & 69 & 11 & 1,412 & 533 & 4.2 & 103 & 6.3 & 134 & 442 & 1.57 & 0.62 \\
\hline & Standard deviation, & 34 & 11 & 1,017 & 181 & 1.9 & 49 & 2.7 & 77 & 305 & 0.37 & 0.23 \\
\hline \multirow[t]{20}{*}{2013} & 1 & 92 & 26 & 2,952 & 622 & 8.6 & 136 & 8.1 & 132 & 1,008 & 2.35 & 0.42 \\
\hline & 2 & 36 & 10 & 1,123 & 736 & 3.2 & 96 & 4.2 & 140 & 216 & 2.49 & 0.44 \\
\hline & 3 & 160 & 36 & 767 & 364 & 4.2 & 136 & 4.2 & 120 & 272 & 2.01 & 0.95 \\
\hline & 4 & 78 & 7.30 & 1,836 & 602 & 5.5 & 176 & 4.4 & 226 & 400 & 1.75 & 0.49 \\
\hline & 5 & 36 & 0.36 & 1,368 & 622 & 3.6 & 102 & 8.2 & 170 & 320 & 2.54 & 0.97 \\
\hline & 6 & 43 & 10 & 572 & 508 & 3.4 & 120 & 4.4 & 38 & 392 & 1.63 & 0.48 \\
\hline & 7 & 128 & 33 & 648 & 508 & 3.4 & 48 & 4.1 & 106 & 248 & 2.57 & 1.17 \\
\hline & 8 & 85 & 5.15 & 547 & 1,057 & 3.6 & 78 & 4.4 & 68 & 296 & 1.88 & 0.80 \\
\hline & 9 & 43 & 3.61 & 328 & 789 & 2.6 & 72 & 4.4 & 98 & 152 & 2.61 & 1.08 \\
\hline & 10 & 107 & 11 & 2,311 & 364 & 6.2 & 76 & 16 & 142 & 904 & 1.70 & 0.58 \\
\hline & 11 & 57 & 0.05 & 1,188 & 488 & 3.6 & 122 & 4.1 & 58 & 608 & 1.90 & 0.54 \\
\hline & 12 & 53 & 0.84 & 4,716 & 675 & 10.2 & 260 & 8.2 & 396 & 968 & 1.97 & 0.54 \\
\hline & 13 & 78 & 27 & 785 & 830 & 3.4 & 126 & 12 & 142 & 224 & 2.15 & 0.82 \\
\hline & 14 & 46 & 0.30 & 2,549 & 291 & 8.2 & 124 & 12 & 280 & 1,064 & 1.57 & 0.92 \\
\hline & 15 & 46 & 0.79 & 2,246 & 384 & 6.2 & 124 & 8.2 & 192 & 664 & 1.31 & 0.72 \\
\hline & 16 & 50 & 4.87 & 1,476 & 622 & 3.2 & 146 & 4.4 & 200 & 312 & 2.06 & 0.59 \\
\hline & Minimum & 36 & 0.05 & 328 & 291 & 2.6 & 48 & 4.1 & 38 & 152 & 1.31 & 0.42 \\
\hline & Maximum & 160 & 36.1 & 4,716 & 1,057 & 10.2 & 260 & 16 & 396 & 1,064 & 2.61 & 1.17 \\
\hline & Mean & 71 & 11.03 & 1,588 & 591 & 4.9 & 121 & 7.0 & 157 & 503 & 2.03 & 0.72 \\
\hline & Standard deviation, \pm & 36 & 12.34 & 1,153 & 201 & 2.3 & 49 & 3.7 & 90 & 318 & 0.39 & 0.24 \\
\hline
\end{tabular}


Table 2. (Continued.)

\begin{tabular}{|c|c|c|c|c|c|c|c|c|c|c|c|c|}
\hline Year & Serial Number & $\mathrm{Cl}^{-}$ & $\mathrm{NO}_{3}^{-}$ & $\mathrm{SO}_{4}^{2-}$ & $\mathrm{HCO}_{3}^{-}$ & $\mathrm{NH}_{4}^{+}$ & $\mathrm{Na}^{+}$ & $\mathrm{K}^{+}$ & $\mathrm{Mg}^{2+}$ & $\mathrm{Ca}^{2+}$ & $\mathrm{Al}$ & $\mathrm{Fe}$ \\
\hline \multirow[t]{20}{*}{2014} & 1 & 104 & 29 & 3,160 & 700 & 9.3 & 163 & 9.5 & 155 & 1,142 & 2.72 & 0.68 \\
\hline & 2 & 43 & 12.1 & 1,318 & 843 & 3.8 & 115 & 4.7 & 164 & 258 & 2.80 & 0.60 \\
\hline & 3 & 181 & 42 & 900 & 422 & 4.9 & 160 & 6.3 & 141 & 352 & 2.42 & 0.95 \\
\hline & 4 & 88 & 8.8 & 2,103 & 694 & 6.0 & 190 & 4.9 & 268 & 465 & 2.10 & 0.77 \\
\hline & 5 & 47 & 0.4 & 1,605 & 712 & 4.9 & 122 & 9.2 & 194 & 367 & 2.84 & 0.97 \\
\hline & 6 & 56 & 12.1 & 771 & 585 & 5.0 & 144 & 4.9 & 45 & 442 & 1.98 & 0.72 \\
\hline & 7 & 143 & 37 & 760 & 588 & 4.8 & 58 & 4.6 & 124 & 290 & 2.83 & 1.05 \\
\hline & 8 & 96 & 6.2 & 755 & 1,200 & 4.6 & 94 & 5.2 & 80 & 365 & 2.19 & 0.86 \\
\hline & 9 & 55 & 5.2 & 672 & 903 & 3.0 & 86 & 5.3 & 115 & 240 & 2.65 & 0.93 \\
\hline & 10 & 121 & 13.3 & 2,710 & 490 & 6.8 & 91 & 18 & 167 & 1,020 & 2.20 & 0.70 \\
\hline & 11 & 64 & 2 & 1,394 & 571 & 4.0 & 146 & 5.2 & 68 & 686 & 2.09 & 0.68 \\
\hline & 12 & 60 & 1 & 5,491 & 776 & 11.1 & 280 & 9.2 & 448 & 1,080 & 2.78 & 0.88 \\
\hline & 13 & 88 & 30 & 966 & 950 & 4.0 & 151 & 13.7 & 167 & 273 & 2.42 & 0.82 \\
\hline & 14 & 52 & 0.4 & 2,860 & 375 & 9.0 & 140 & 15.8 & 300 & 1,200 & 2.14 & 0.91 \\
\hline & 15 & 54 & 1 & 2,540 & 460 & 6.8 & 149 & 9.2 & 225 & 749 & 1.92 & 0.65 \\
\hline & 16 & 60 & 5.9 & 1,627 & 719 & 4.1 & 155 & 4.9 & 230 & 363 & 2.34 & 0.66 \\
\hline & Minimum & 43 & 0.4 & 672 & 375 & 3.0 & 58 & 4.6 & 45 & 240 & 1.92 & 0.60 \\
\hline & Maximum & 181 & 42 & 5,491 & 1,200 & 11.1 & 280 & 18 & 448 & 1,200 & 2.84 & 1.05 \\
\hline & Mean & 82 & 12.9 & 1,852 & 687 & 5.8 & 140 & 8.2 & 181 & 581 & 2.40 & 0.80 \\
\hline & Standard deviation, \pm & 39 & 13.8 & 1,278 & 217 & 2.3 & 51 & 4.3 & 100 & 347 & 0.33 & 0.14 \\
\hline \multirow[t]{20}{*}{2015} & 1 & 98 & 28 & 3,110 & 655 & 8.8 & 151 & 9.0 & 145 & 1,075 & 2.60 & 0.70 \\
\hline & 2 & 40 & 11.1 & 1,241 & 795 & 3.6 & 107 & 4.5 & 154 & 243 & 2.90 & 0.60 \\
\hline & 3 & 166 & 38 & 848 & 390 & 4.6 & 151 & 6.0 & 132 & 312 & 2.12 & 1.00 \\
\hline & 4 & 83 & 8.1 & 1,980 & 650 & 5.7 & 180 & 4.7 & 250 & 425 & 1.85 & 0.70 \\
\hline & 5 & 40 & 0.4 & 1,512 & 672 & 4.6 & 113 & 8.7 & 181 & 340 & 2.72 & 1.00 \\
\hline & 6 & 53 & 11.1 & 632 & 552 & 4.7 & 133 & 4.7 & 42 & 416 & 1.72 & 0.60 \\
\hline & 7 & 136 & 36 & 716 & 555 & 4.5 & 53 & 4.4 & 116 & 263 & 2.71 & 1.10 \\
\hline & 8 & 92 & 5.7 & 604 & 1,130 & 4.3 & 87 & 4.7 & 75 & 344 & 2.10 & 0.80 \\
\hline & 9 & 52 & 4 & 362 & 852 & 2.8 & 80 & 5.0 & 108 & 198 & 2.75 & 1.00 \\
\hline & 10 & 113 & 12.2 & 2,580 & 450 & 6.4 & 84 & 17.1 & 156 & 960 & 1.90 & 0.60 \\
\hline & 11 & 63 & 1 & 1,313 & 539 & 3.8 & 135 & 4.4 & 64 & 646 & 2.00 & 0.60 \\
\hline & 12 & 57 & 0.9 & 5,170 & 732 & 10.5 & 270 & 8.7 & 430 & 1,034 & 2.08 & 0.80 \\
\hline & 13 & 83 & 30 & 910 & 896 & 3.8 & 140 & 13.0 & 156 & 257 & 2.27 & 0.80 \\
\hline & 14 & 49 & 0.3 & 2,770 & 340 & 8.5 & 130 & 15.0 & 295 & 1,135 & 1.66 & 1.00 \\
\hline & 15 & 51 & 0.9 & 2,410 & 415 & 6.4 & 138 & 8.7 & 211 & 705 & 1.60 & 0.70 \\
\hline & 16 & 56 & 5.4 & 1,532 & 678 & 3.8 & 150 & 4.7 & 215 & 342 & 2.20 & 0.60 \\
\hline & Minimum & 40 & 0.3 & 362 & 340 & 2.8 & 53 & 4.4 & 42 & 198 & 1.60 & 0.60 \\
\hline & Maximum & 166 & 38 & 5,170 & 1,130 & 10.5 & 270 & 17.1 & 430 & 1,135 & 2.90 & 1.10 \\
\hline & Mean & 77 & 12 & 1,731 & 644 & 5.4 & 131 & 7.7 & 171 & 543 & 2.20 & 0.80 \\
\hline & Standard deviation, & 36 & 13 & 1,249 & 208 & 2.2 & 50 & 4.1 & 96 & 333 & 0.40 & 0.20 \\
\hline \multirow[t]{20}{*}{2016} & 1 & 110 & 31 & 3,190 & 749 & 9.8 & 169 & 9.9 & 163 & 1,210 & 2.80 & 0.83 \\
\hline & 2 & 45 & 12.9 & 1,406 & 890 & 4.0 & 122 & 4.9 & 173 & 279 & 3.10 & 0.86 \\
\hline & 3 & 194 & 45 & 960 & 452 & 5.1 & 170 & 6.6 & 149 & 380 & 2.49 & 0.93 \\
\hline & 4 & 98 & 9.5 & 2,244 & 743 & 6.3 & 201 & 5.2 & 282 & 502 & 2.16 & 0.82 \\
\hline & 5 & 45 & 0.5 & 1,713 & 762 & 5.1 & 130 & 9.6 & 204 & 396 & 2.93 & 0.95 \\
\hline & 6 & 54 & 12.9 & 823 & 626 & 5.2 & 152 & 5.2 & 47 & 477 & 2.04 & 0.92 \\
\hline & 7 & 158 & 38 & 811 & 629 & 5.0 & 61 & 4.8 & 131 & 313 & 3.12 & 1.00 \\
\hline & 8 & 102 & 6.7 & 806 & 1,250 & 4.8 & 99 & 5.4 & 84 & 395 & 2.26 & 0.89 \\
\hline & 9 & 54 & 5.6 & 913 & 966 & 3.5 & 91 & 5.5 & 121 & 259 & 2.73 & 0.90 \\
\hline & 10 & 130 & 14.2 & 2,992 & 524 & 7.1 & 97 & 19 & 176 & 1,070 & 2.27 & 0.88 \\
\hline & 11 & 71 & 2.1 & 1,488 & 611 & 4.2 & 155 & 5.4 & 88 & 741 & 2.16 & 0.93 \\
\hline & 12 & 66 & 1.1 & 5,580 & 830 & 11.7 & 295 & 9.6 & 473 & 1,140 & 2.90 & 0.90 \\
\hline & 13 & 94 & 31 & 1,120 & 1,005 & 4.2 & 160 & 15 & 176 & 295 & 2.49 & 0.82 \\
\hline & 14 & 58 & 0.4 & 2,960 & 401 & 9.5 & 149 & 17 & 317 & 1,230 & 2.20 & 0.96 \\
\hline & 15 & 58 & 1.0 & 2,679 & 492 & 7.1 & 158 & 10 & 238 & 780 & 1.98 & 0.86 \\
\hline & 16 & 63 & 6.3 & 1,720 & 769 & 4.3 & 167 & 5.2 & 243 & 392 & 2.41 & 0.87 \\
\hline & Minimum & 45 & 0.4 & 806 & 401 & 3.5 & 61 & 4.8 & 47 & 259 & 2.00 & 0.80 \\
\hline & Maximum & 194 & 45 & 5,580 & 1,250 & 11.7 & 295 & 19.0 & 473 & 1,230 & 3.10 & 1.00 \\
\hline & Mean & 88 & 14 & 1,963 & 731 & 6.1 & 149 & 8.6 & 192 & 616 & 2.50 & 0.90 \\
\hline & Standard deviation, \pm & 43 & 15 & 1,283 & 225 & 2.4 & 53 & 4.6 & 104 & 358 & 0.40 & 0.10 \\
\hline
\end{tabular}




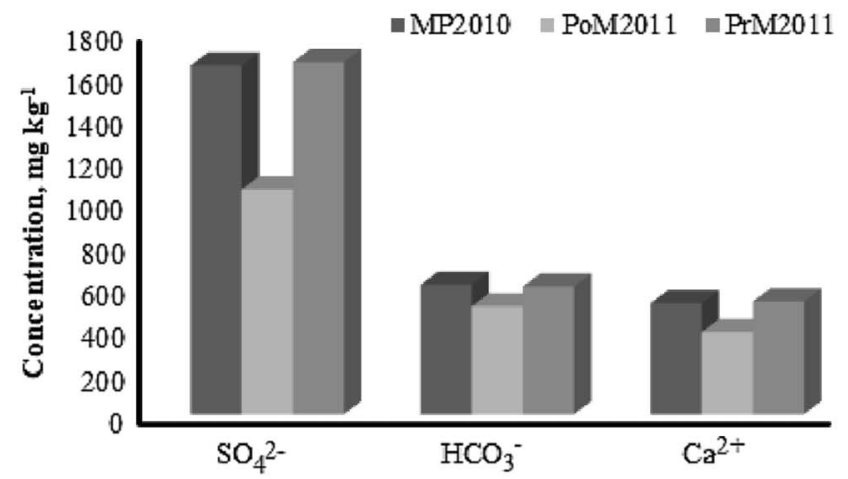

(a)

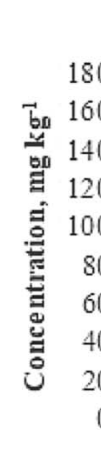

(b)

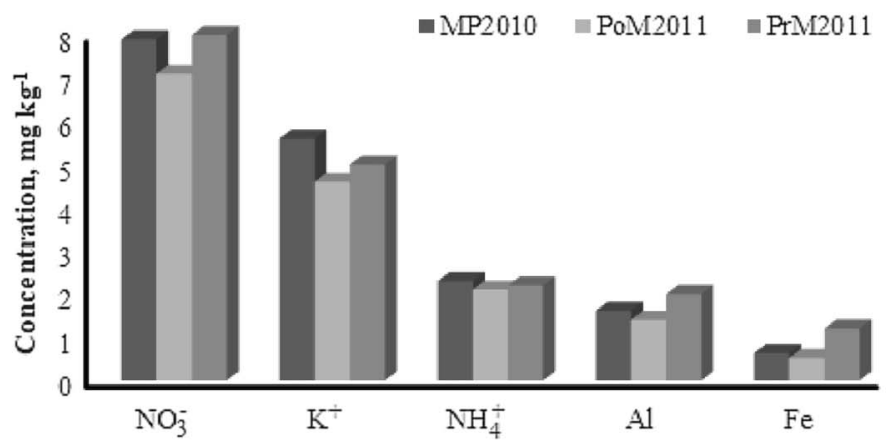

(c)

Ion

Fig. 2. Seasonal variations in concentrations of chemical species during monsoon, premonsoon, and postmonsoon periods: (a) $\mathrm{SO}_{4}^{2-}$, $\mathrm{HCO}_{3}^{-}$, and $\mathrm{Ca}^{2+}$; (b) $\mathrm{Mg}^{2+}, \mathrm{Na}^{+}$, and $\mathrm{Cl}^{-}$; and (c) $\mathrm{NO}_{3}^{-}, \mathrm{K}^{+}, \mathrm{NH}_{4}^{+}, \mathrm{Al}$, and $\mathrm{Fe}$.

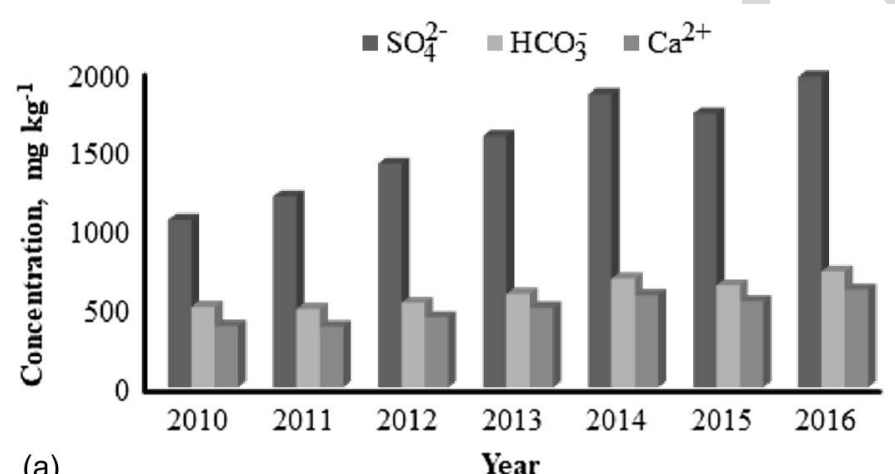

(a)
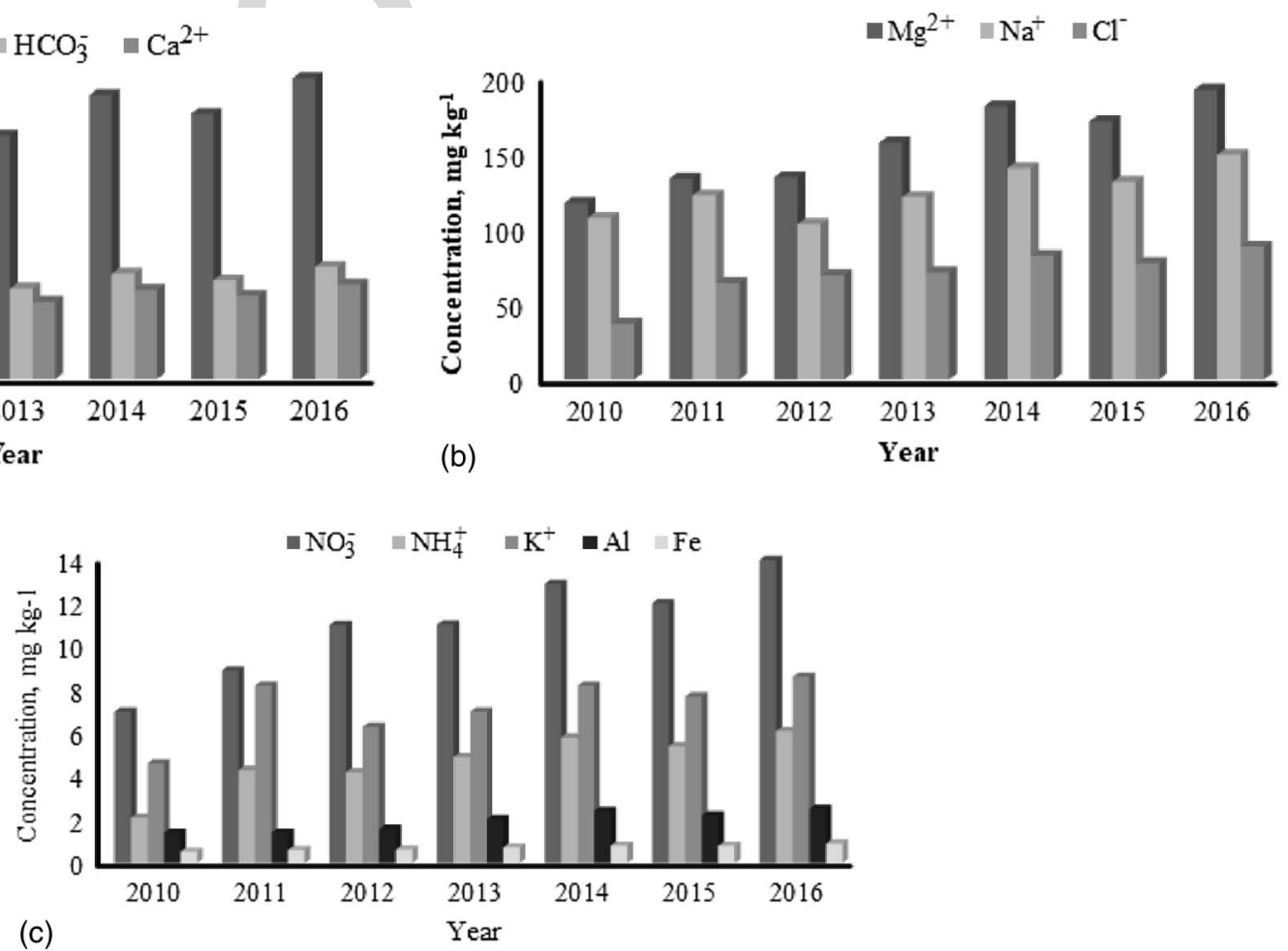

Fig. 3. Temporal variations in concentration of chemical species during postmonsoon period, 2010-2016: (a) $\mathrm{SO}_{4}^{2-}, \mathrm{HCO}_{3}^{-}$, and $\mathrm{Ca}^{2+}$; (b) $\mathrm{Mg}^{2+}$, $\mathrm{Na}^{+}$, and $\mathrm{Cl}^{-}$; and (c) $\mathrm{NO}_{3}^{-}, \mathrm{K}^{+}, \mathrm{NH}_{4}^{+}, \mathrm{Al}$, and $\mathrm{Fe}$. 


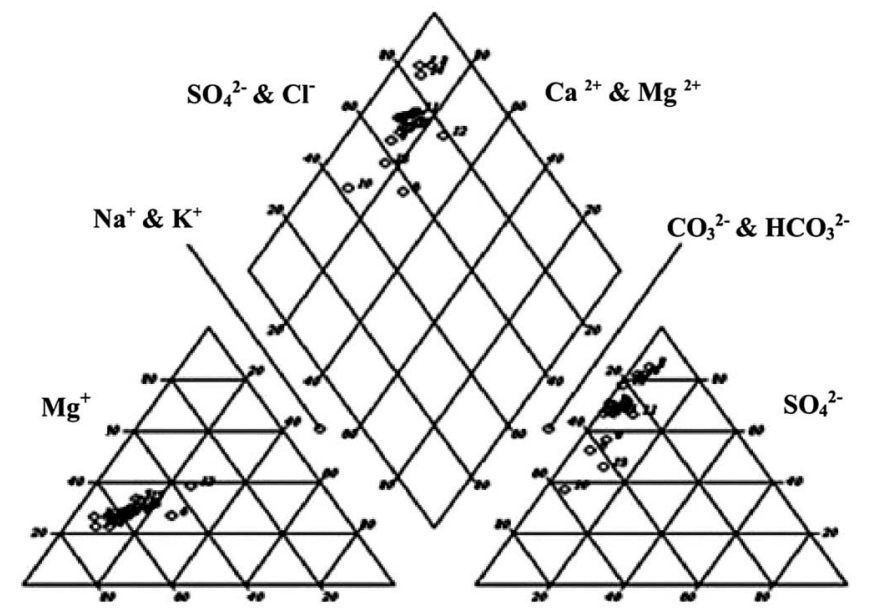

Fig. 4. Piper diagrams of groundwater in postmonsoon period, 2010.

samples from the tube well in Location 12. Nitrate concentration ranged from 1.2 to $18 \mathrm{mg} \mathrm{L}^{-1}$, below the limit of $45 \mathrm{mg} \mathrm{L}^{-1}$ (BIS 2009; WHO 2011).

Aluminum and iron are the most abundant metallic elements of the Earth's outer crust, so their presence in the groundwater samples originate from a variety of mineral sources. Al and Fe concentrations ranged respectively from 1.1 to $1.7 \mathrm{mg} \mathrm{L}^{-1}$ and from 0.32 to $0.90 \mathrm{mg} \mathrm{L}^{-1}$, exceeding the recommended limits of 0.03 and $0.3 \mathrm{mg} \mathrm{L}^{-1}$ for all tube wells (BIS 2009; WHO 2011).

The Eanion/ $\Sigma$ cation ratio, expressed in terms of equivalent concentrations, was found to vary between 0.89 and 1.27 , with a mean value of $1.02 \pm 0.04 . \mathrm{SO}_{4}^{2-}$ was the dominating species, moderately/well correlated with $\mathrm{Na}^{+}, \mathrm{Mg}^{2+}, \mathrm{Ca}^{2+}, \mathrm{Al}$, and $\mathrm{Fe}$ (with $r$ values in the 0.68-0.91 range). On average, across the 16 tube wells, ions in the water (in terms of equivalent concentration) appeared in the following decreasing order: $\mathrm{SO}_{4}^{2-}>\mathrm{Ca}^{2+}>$ $\mathrm{HCO}_{3}^{-}>\mathrm{Mg}^{2+}>\mathrm{Na}^{+}>\mathrm{Cl}^{-}>\mathrm{NO}_{3}^{-}>\mathrm{K}^{+}>\mathrm{NH}_{4}^{+}$.

\section{Seasonal and Temporal Variations}

The seasonal variations in the concentration of the ions are depicted in Fig. 2. The concentration of all the ions (except $\mathrm{NH}_{4}^{+}$and $\mathrm{NO}_{3}^{-}$) was found to decrease during the monsoon period due to high rainfall (approximately $100 \mathrm{~cm}$ ), while their concentrations (except for $\mathrm{NH}_{4}^{+}, \mathrm{NO}_{3}^{-}$, and $\mathrm{Cl}^{-}$) notably increased in the premonsoon period owing to an increase in water temperature (approximately $26^{\circ} \mathrm{C}-30^{\circ} \mathrm{C}$ ) and reduction of water levels (approximately $20-40 \mathrm{~m}$ ). An increase in the concentration of ions was also observed over a 7-year period, from 2010 to 2016 (Fig. 3), tentatively ascribed to a gradual increase in the extraction of groundwater for domestic and agricultural purposes.

\section{Water Classification}

The groundwater samples were classified into two types: $\mathrm{Ca}-\mathrm{Mg}-\mathrm{SO}_{4}-\mathrm{HCO}_{3}$ and $\mathrm{Ca}-\mathrm{Mg}-\mathrm{Na}-\mathrm{SO}_{4}-\mathrm{HCO}_{3}$ in accordance with the piper diagrams (Fig. 4). The dominant type, $\mathrm{Ca}-\mathrm{Mg}-\mathrm{SO}_{4}-\mathrm{HCO}_{3}$, showed effects of gypsum and dolomite mineral dissolution. Enrichment in $\mathrm{Na}^{+}$indicated dissolution of sodium-containing minerals in the aquifer.

The dendrograms generated by cluster analysis also presented two groups of water samples in the studied area (Fig. 5). The two clusters described the underlying process that led to spatial variations. Cluster $1(n=10)$, with two subgroups, contained

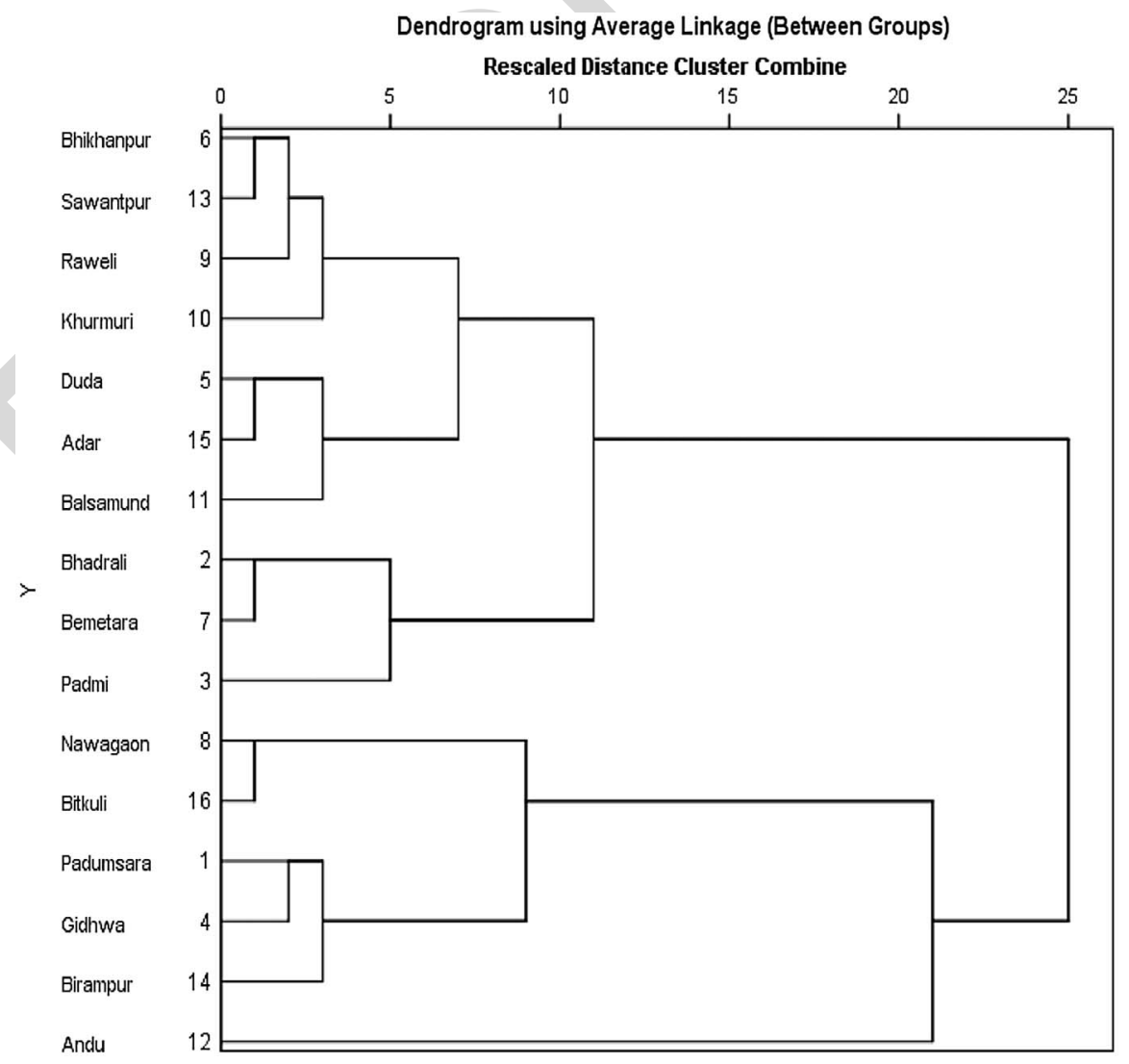

Fig. 5. Dendrogram of groundwater samples in postmonsoon period, 2010. 
groundwater samples 6, 13, 9-10, 5, 15, 11, 2, 7, and 3, while Cluster $2(n=6)$ contained groundwater samples $8,16,1,14$, and 12 . Parameters such as EC, TDS, $\mathrm{SO}_{4}^{2-}, \mathrm{HCO}_{3}^{-}, \mathrm{Na}^{+}, \mathrm{Mg}^{2+}$, $\mathrm{Ca}^{2+}, \mathrm{Al}$, and $\mathrm{Fe}$ were the discriminating species. The mean values of EC, TDS, $\mathrm{SO}_{4}^{2-}, \mathrm{HCO}_{3}^{-}, \mathrm{Na}^{+}, \mathrm{Mg}^{2+}, \mathrm{Ca}^{2+}, \mathrm{Al}$, and Fe were at least 1.8 times higher in the water from locations belonging to the Cluster 2 group.

\section{Water Quality Assessment}

The values of TDS, TH, TA, $\mathrm{SO}_{4}^{2-}, \mathrm{Mg}^{2+}, \mathrm{Ca}^{2+}, \mathrm{Al}$, and $\mathrm{Fe}$ of the groundwater samples under study were higher than the recommended values of 500, 300, 120, 150, 30, 75, 0.03, and $0.3 \mathrm{mg} \mathrm{L}^{-1}$, respectively, and the EC value was also very high. The WQI value of the water samples ranged from 187 to 806, with a mean value of $406 \pm 82$, clearly indicating that the groundwater of the Bemetara area would be unsuitable for drinking.

The SAR, KR, SH, MH, and PI values of the groundwater of the studied area were in the following ranges: $0.7-5.1,6-35,4.2 \%$ $14.0 \%, 25 \%-52 \%$, and $13 \%-39 \%$, with mean values of $1.7 \% \pm$ $1.0 \%, 16 \pm 8,8.3 \% \pm 3.2 \%, 33 \% \pm 7 \%$, and $23 \% \pm 7 \%$, respectively (Table 3). Generally, water with $\mathrm{MH}<50 \%$, SAR $<10$, and $\mathrm{KR}<1$ is considered suitable for irrigation (WHO 2011; Richard 1954; Kelley 1940; Wilcox 1955). Further, the SH value of the water from all tube wells was $<20 \%$, which indicates that the water would be suitable for irrigation purposes (Wilcox 1955). However, the PI value in the water of $63 \%$ of the tube wells was $<25 \%$, indicating that it would not be adequate for irrigation (Doneen 1964). In short, the groundwater of the Bemetara area may be used for the irrigation of sulfate-insensitive plants.

\section{Source Analysis}

Factor analysis was applied to the data set obtained from the groundwater samples from the 16 selected locations in the postmonsoon period. The correlation matrix of variables was generated, factors were extracted by principal components analysis, and a normalized Varimax rotation was applied (Table 4). Four factors accounted for $83.04 \%$ of the total variance. Factor 1 had high loading values for $\mathrm{EC}, \mathrm{TDS}, \mathrm{SO}_{4}^{2-}, \mathrm{Na}^{+}, \mathrm{Mg}^{2+}, \mathrm{Ca}^{2+}, \mathrm{Al}$, and $\mathrm{Fe}$ variables, explained $47.22 \%$ of the total variance, and would represent the mineralization of groundwater. Factor 2 was strongly correlated with the physical parameter depth and negatively correlated with variable age. It accounted for $15.22 \%$ of total variance

Table 3. Water quality indices of groundwater during January 2010

\begin{tabular}{lcccccc}
\hline Serial Number & WQI & SAR & KR & SH & PI & MH \\
\hline 1 & 594 & 1.8 & 13 & 14 & 19 & 26 \\
2 & 376 & 1.6 & 16 & 7.8 & 23 & 31 \\
3 & 422 & 1.5 & 13 & 9.2 & 21 & 29 \\
4 & 548 & 1.6 & 12 & 14 & 18 & 31 \\
5 & 325 & 1.4 & 15 & 7 & 24 & 39 \\
6 & 251 & 2.6 & 34 & 7.3 & 39 & 37 \\
7 & 331 & 0.7 & 6 & 4.2 & 13 & 28 \\
8 & 429 & 1.2 & 10 & 4.3 & 14 & 30 \\
9 & 193 & 1.1 & 15 & 5.9 & 27 & 29 \\
10 & 187 & 0.8 & 11 & 7.8 & 31 & 30 \\
11 & 385 & 1.7 & 16 & 5.6 & 22 & 35 \\
12 & 806 & 5.1 & 35 & 13 & 31 & 52 \\
13 & 249 & 1.5 & 19 & 7.5 & 30 & 36 \\
14 & 554 & 1.2 & 9 & 7.2 & 14 & 25 \\
15 & 314 & 1.5 & 17 & 6.5 & 26 & 39 \\
16 & 524 & 1.8 & 15 & 12 & 22 & 33 \\
\hline
\end{tabular}

Table 4. Factor loading matrix and total variance explained during January 2010

\begin{tabular}{lrrrr}
\hline Variable & \multicolumn{1}{c}{ I } & \multicolumn{1}{c}{ II } & \multicolumn{1}{c}{ III } & \multicolumn{1}{c}{ IV } \\
\hline Depth & 0.14 & $\mathbf{0 . 9 4}$ & 0.02 & 0.09 \\
Age & -0.17 & $-\mathbf{0 . 9 0}$ & -0.17 & -0.08 \\
$\mathrm{pH}$ & 0.09 & 0.20 & 0.42 & 0.62 \\
$\mathrm{EC}$ & $\mathbf{0 . 9 6}$ & 0.21 & 0.02 & 0.16 \\
$\mathrm{TDS}$ & $\mathbf{0 . 9 6}$ & 0.22 & 0.02 & 0.17 \\
$\mathrm{Cl}^{-}$ & -0.16 & 0.12 & 0.13 & $-\mathbf{0 . 9 4}$ \\
$\mathrm{NO}_{3}^{-}$ & -0.21 & 0.07 & $\mathbf{0 . 7 6}$ & -0.03 \\
$\mathrm{SO}_{4}^{2-}$ & $\mathbf{0 . 9 6}$ & 0.14 & -0.11 & 0.01 \\
$\mathrm{HCO}_{3}^{-}$ & 0.54 & 0.38 & -0.20 & 0.51 \\
$\mathrm{NH}_{4}^{+}$ & 0.14 & 0.02 & $\mathbf{0 . 8 4}$ & -0.03 \\
$\mathrm{Na}^{+}$ & $\mathbf{0 . 8 0}$ & -0.08 & 0.28 & 0.25 \\
$\mathrm{~K}^{+}$ & 0.04 & 0.54 & -0.09 & 0.67 \\
$\mathrm{Mg}^{2+}$ & $\mathbf{0 . 9 1}$ & 0.08 & 0.24 & 0.15 \\
$\mathrm{Ca}^{2+}$ & $\mathbf{0 . 8 1}$ & 0.32 & -0.30 & -0.06 \\
$\mathrm{Al}^{2+}$ & $\mathbf{0 . 8 4}$ & 0.33 & -0.18 & 0.17 \\
$\mathrm{Fe}_{\text {Eigenvalue }}$ & $\mathbf{0 . 8 2}$ & -0.23 & -0.09 & -0.03 \\
$\mathrm{Total}^{-}$variance $(\%)$ & 6.71 & 2.57 & 1.84 & 2.16 \\
$\mathrm{Cumulative} \mathrm{value}(\%)^{2}$ & 47.22 & 15.22 & 11.20 & 9.40 \\
\hline & 47.22 & 62.44 & 73.64 & 83.04 \\
\hline
\end{tabular}

Note: Significant loading value $(>0.70)$ in bold, at $p<0.05$.

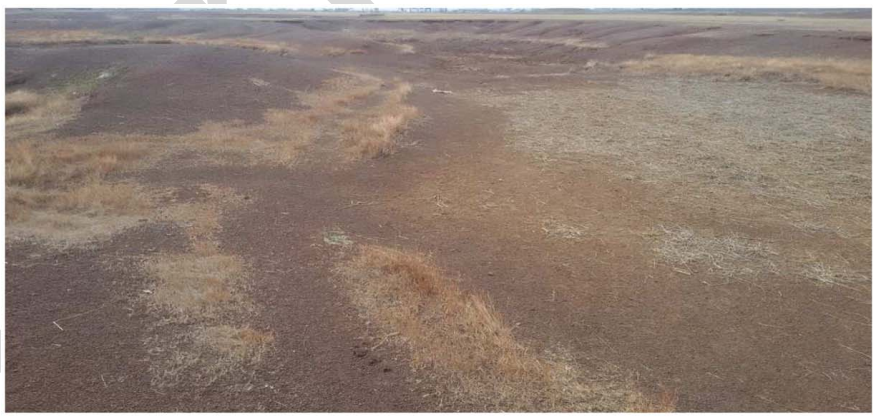

(a)

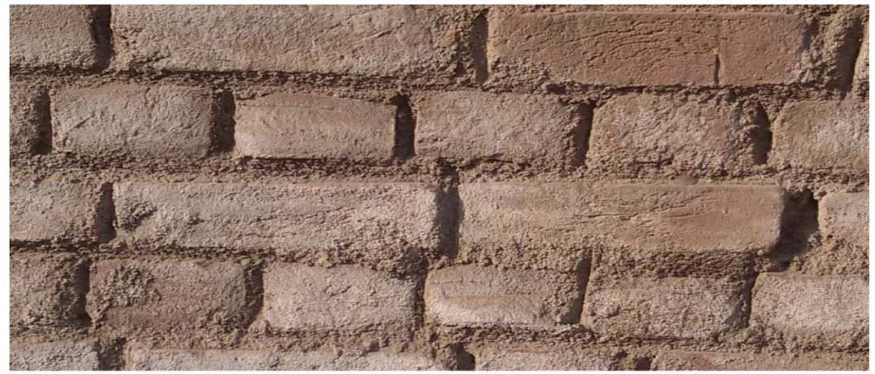

(b)

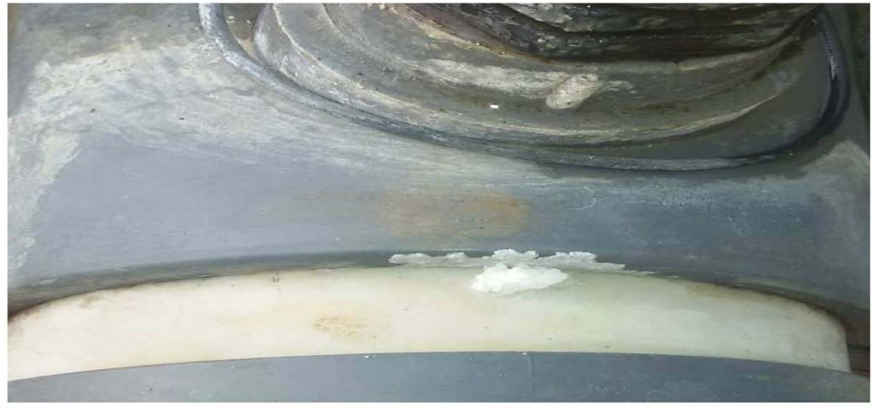

(c)

Fig. 6. Environmental hazards: (a) bare and scalded land; (b) white patches in building bricks; and (c) rusting of water supply pipes. (Images by Suryakant Chakradhari.) 


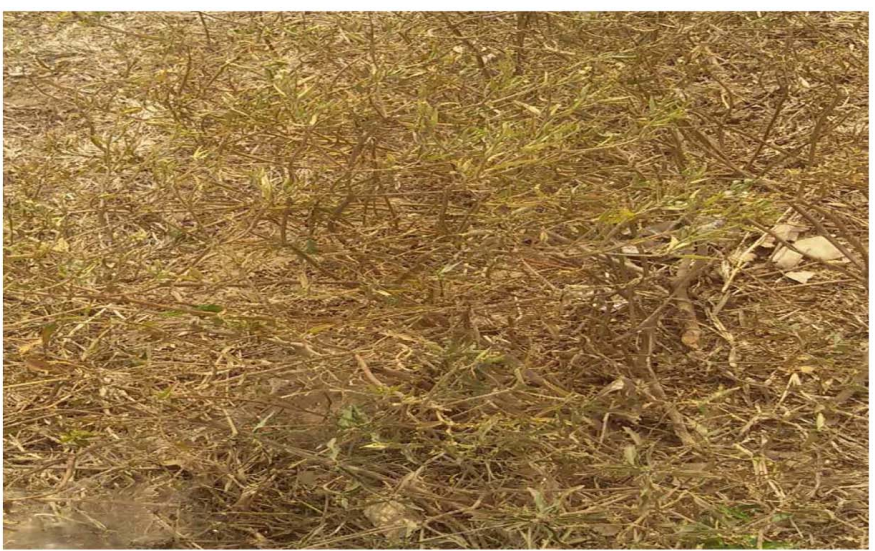

(a)

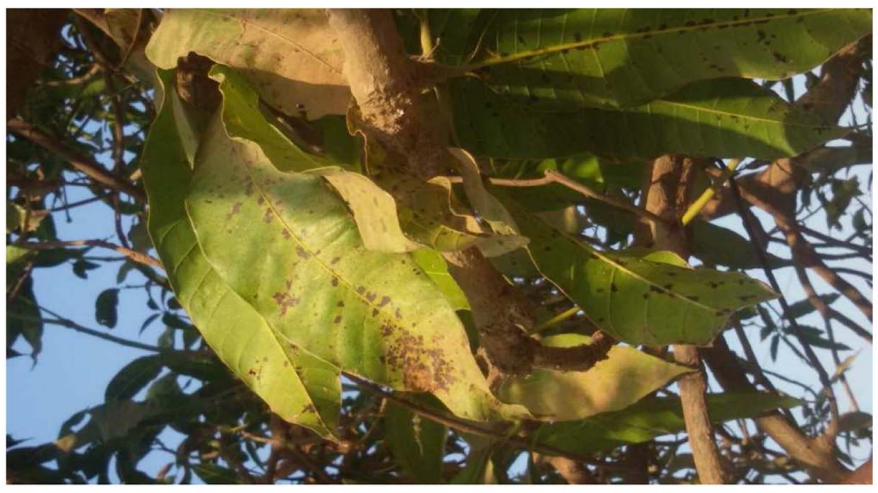

(b)

Fig. 7. Chlorosis of leaves: (a) pigeon pea (Cajanus cajan); and (b) mango leaves. (Images by Suryakant Chakradhari.)

and may be ascribed to the influence of deep groundwater on the water chemical composition. Factor 3 was related to $\mathrm{NO}_{3}^{-}$and $\mathrm{NH}_{4}^{+}$ and accounted for $11.20 \%$ of total variance. It may represent the impact of agricultural practices (fertilization). Factor 4 accounted for $9.40 \%$ of total variance and was associated with $\mathrm{Cl}^{-}$, tentatively representing the impact of irrigation flow return on groundwater quality.

\section{Environmental Hazards}

Environmental hazards associated with the use of groundwater in gypsum-contaminated areas include, for example, scalding of land; the appearance of a white crust on the soil surface; negative impacts on crop health; a decline in grass, shrubs, and trees; and the corrosion of sewer pipes and buildings (Ayers and Westcot 1985; Dunlop et al. 2005; UNEP 2008).

Gypsum deposition on soil surface due to irrigation increases osmotic pressure, resulting in physiological drought, with negative impacts on plant growth and yield. The surface soil in many locations in the area of study were bared and scalded, as shown in Fig. 6(a). Gypsum also attacks zinc and iron (steel) in combination with moisture. A white salt crust on brickwork and rusting of pipes due to the deposition of gypsum salt were also observed [Figs. 6(b and c)].

Symptoms on crops (e.g., chlorosis in leaves or browning of roots) are evident for sensitive plants such as barley, cucumber, lemons, lettuce, mango, pumpkin, sunflower, pomegranate, pigeon pea, potatoes, rice, soybean, and watermelon (Fig. 7). In contrast, higher yields are obtained for sulfate-tolerant plants (e.g., beans,

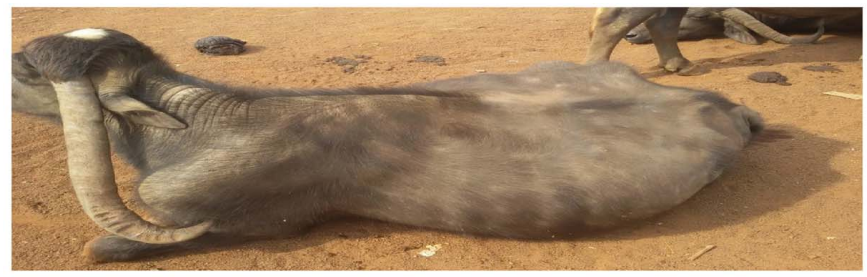

(a)

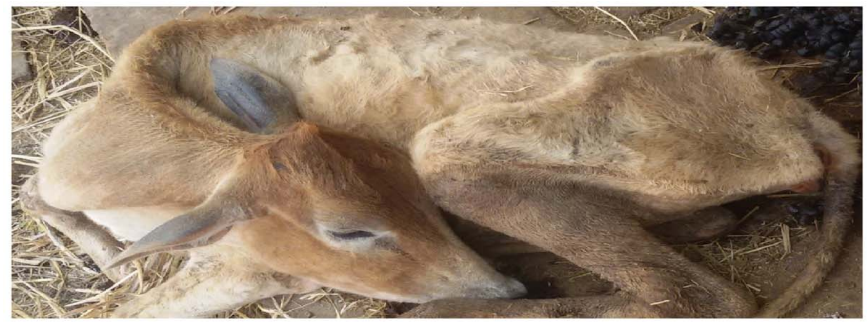

(b)

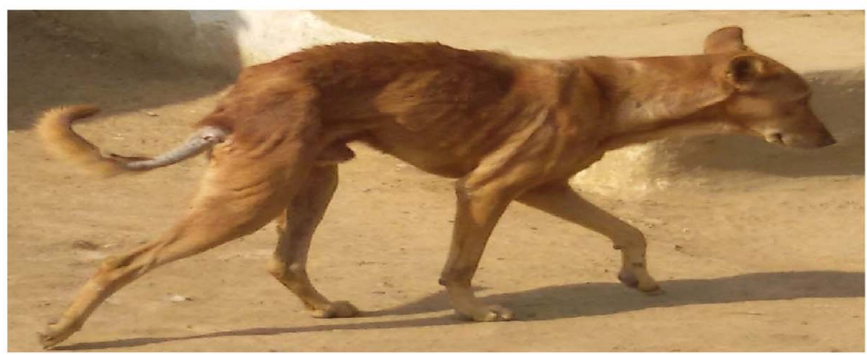

(c)

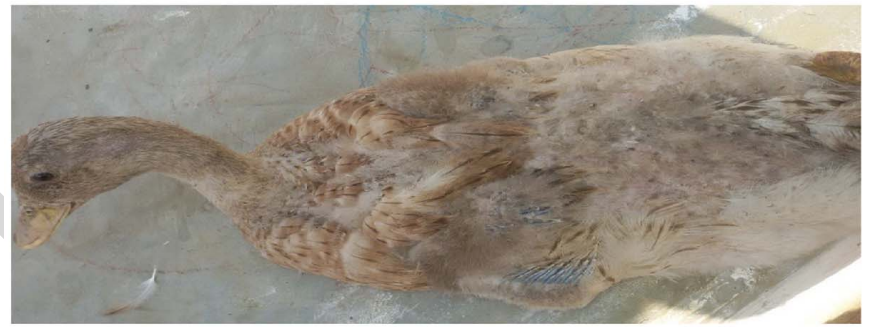

(d)

Fig. 8. Adverse health hazard in animals and aquatics: (a) buffalo; (b) cow; (c) dog; and (d) duck. (Images by Suryakant Chakradhari.)

cabbage, carrot, cauliflower, mustard, garlic, ginger, green beans, lentils, onion, peanuts, pineapple, radish, and spinach).

Regarding negative impacts on animal health, severe chronic diarrhea and, in some cases, death, are frequent in the area due to the high levels of sulfate in the drinking water. Examples for several domestic animals are shown in Fig. 8.

\section{Conclusions}

Groundwater in the Bemetara area of central India is very hard due to its high salt content. Physicochemical characteristics of water were monitored both on a seasonal basis and over a 7-year period, and the concentrations of $\mathrm{Ca}^{2+}\left(169-660 \mathrm{mg} \mathrm{L}^{-1}\right)$, $\mathrm{Mg}^{2+}$ (43-341 mg L$\left.{ }^{-1}\right), \mathrm{SO}_{4}^{2-}\left(254-2,330 \mathrm{mg} \mathrm{L}^{-1}\right), \quad \mathrm{Al}(1.1-$ $\left.1.7 \mathrm{mg} \mathrm{L}^{-1}\right)$, and $\mathrm{Fe}\left(0.32-0.90 \mathrm{mg} \mathrm{L}^{-1}\right)$ were much higher than the recommended values. Very high $\mathrm{Na}^{+}$concentrations (up to $437 \mathrm{mg} \mathrm{L}^{-1}$ ) were also detected in particular locations. Concentrations were found to increase in the premonsoon season and over time, due to the gradual increase in the extraction of groundwater for domestic and agricultural purposes. Based on the calculated 
water quality indices, the groundwater may be deemed suitable for irrigation in some cases, but would be clearly unsuitable for drinking purposes. Negative impacts on animals, crops, and materials were observed. Avoiding overirrigation and plantation of highsulfate-tolerant plants are suggested as the most promising approaches for sustainable agricultural development in this region.

\section{Acknowledgments}

Nohar Singh Dahariya and Khageshwar Singh Patel are grateful to the University Grant Commission, New Delhi, for the award of the Rajiv Gandhi Fellowship and Basic Science Research fellowships.

\section{References}

APHA. 2005. Standard methods for the examination of water and wastewater. 21st ed. Washington, DC: APHA.

Atasoy, A. D., and M. I. Yesilnacar. 2010. "Effect of high sulfate concentration on the corrosivity: A case study from groundwater in Harran plain, Turkey." Environ. Monit. Assess. 166 (1-4): 595-607. https://doi .org/10.1007/s10661-009-1026-2.

Ayers, R. S., and D. W. Westcot. 1985. "Water quality for agriculture, food and agriculture." Accessed January 19, 2013. http://www.fao.org /DOCReP/003/T0234e/T0234e00.htm.

Backer, L. C. 2008. "Assessing the acute gastrointestinal effects of ingesting naturally occurring, high levels of sulfate in drinking water." Crit. Rev. Clin. Lab. Sci. 37 (4): 389-400. https://doi.org/10.1080 /10408360091174259.

Bhandary, H., C. Sabarathinam, and A. Al-Khalid. 2018. "Occurrence of hypersaline groundwater along the coastal aquifers of Kuwait." Desalination 436 (Jun): 15-27. https://doi.org/10.1016/j.desal.2018 .02 .004 .

BIS (Bureau of Indian Standards). 2009. "Indian standard drinking water specifications." Accessed January 19, 2013. http://bis.org.in/sf/fad /FAD25\%282047\%29C.pdf.

Burgess, B. A., K. L. Lohmann, and B. R. Blakley. 2010. "Excessive sulfate and poor water quality as a cause of sudden deaths and an outbreak of diarrhea in Horses." Can. Vet. J. 51 (3): 277-282.

Davis, S. N., and R. J. M. Wiest de. 1966. Hydrogeology. New York: Wiley.

Doneen, L. D. 1964. Water quality for agriculture. Davis, CA: Univ. of California.

Dunlop, J., G. McGregor, and N. Horrigan. 2005. "Potential impacts of salinity and turbidity in riverine ecosystems." In The national action plan for salinity and water quality. Queensland, Australia: SBN.

Greenwood, N. N., and A. Earnshaw. 1984. Chemistry of the elements. Oxford, UK: Pergamon Press.

Hajalilou, B., and F. Khaleghi. 2009. "Investigation of hydro geochemical factors and groundwater quality assessment in Marand municipality, Northwest of Iran: A multivariate statistical approach.” J. Food Agric. Environ. 7 (3-4): 930-937.

Han, D., X. Song, J. Matthew, and M. J. Currell. 2016. "Identification of anthropogenic and natural inputs of sulfate into a karstic coastal groundwater system in northeast China: Evidence from major ions, $\delta^{13} \mathrm{CDIC}$ and $\delta^{1334}$ SSO4." Hydrol. Earth Syst. Sci. 20 (5): 1983-1999. https://doi .org/10.5194/hess-20-1983-2016.
Horst, A., J. Mahlknecht, M. A. López-Zavala, and B. Mayer. 2011. "The origin of salinity and sulphate contamination of groundwater in the Colima state, Mexico, Constrained by Stable Isotopes." Environ. Earth Sci. 64 (7): 1931-1941. https://doi.org/10.1007/s12665-011-1008-x.

Kelley, W. P. 1940. "Permissible composition and concentration of irrigated waters." Proc. Am. Soc. Civ. Eng. 66: 607-613.

Kukillaya, J. P., K. Padmanabhan, and K. Radhakrishnan. 2004. "Occurrence of brackish groundwater in fractured hard rock aquifers of Puzhakkal-Avanur area in Thrissur, Kerala." J. Geol. Soc. Ind. 64 (1): 32-42.

Lorente, S., M. -P. Yssorche-Cubaynes, and J. Auger. 2011. "Sulfate transfer though concrete: Migration and diffusion results." Cem. Concr. Compos. 33 (7): 735-741. https://10.1016/j.cemconcomp.2011.05.001.

MDH (Minnesota Dept. of Health). 2008. "Sulfate in well water." Accessed January 19, 2013. www.health.state.mn.us/divs/eh/wells/waterquality /sulfate.html.

Mubarak, N., I. Hussain, M. Faisal, T. Hussain, M. Yousaf Shad, N. M. AbdEl-Salam, and J. Shabbir. 2015. "Spatial distribution of sulfate concentration in groundwater of South-Punjab, Pakistan." Water Qual. Exposure Health 7 (4): 503-513. https://doi.org/10.1007/s12403-015 -0165-7.

Nielsen, D. M., and G. Nielsen. 2006. The essential handbook of groundwater sampling. Boca Raton, FL: CRC Press.

Nollet Leo, M. L., and S. P. De Gelder Leen. 2007. Handbook of water analysis. 2nd ed. Boca Raton, FL: CRC Press.

Pradhan, B. 2014. "Corrosion behavior of steel reinforcement in concrete exposed to composite chloride-sulfate environment." Constr. Build. Mater. 72 (Dec): 398-410. https://doi.org/10.1016/j.conbuildmat.2014.09.026.

Razowska-Jaworek, L. 2014. Calcium and magnesium in groundwater: occurrence and significance for human health. 1st ed. Boca Raton, FL: CRC Press.

Richards, L. A. 1954. Diagnosis and improvement of saline and alkali soils. Washington, DC: USDA.

Seller, L. E., and L. W. Canter. 1980. Sulfates in surface and groundwater. Norman, OK: National Center for Ground Water Research.

Shrestha, S., and F. Kazama. 2007. "Assessment of surface water quality using Multivariate Statistical Techniques: A case study of the fuji river basin, Japan." Environ. Model. Software 22 (4): 464-475. https://doi .org/10.1016/j.envsoft.2006.02.001.

Stanton, J. S., et al. 2017. "Brackish groundwater in the United States." US Geol. Surv. Prof. 185. https://doi.org/10.3133/pp1833.

UNEP (United Nations Environment Programme). 2008. Water quality for ecosystem and human health. 2nd ed. Ontario, Canada: UNEP United Nations Environment Programme.

van Weert, F., J. van der Gun, and J. Reckman. 2009. "Global overview of saline groundwater occurrence and genesis, Utrecht, NL." Accessed January 19, 2013. http://www.un-igrac.org/sites/default/files/resources /files/Global\%20Overview\%20of\%20Saline\%20Groundwater\%20 Occurences\%20and\%20Genesis.pdf.

WHO. 2011. Guidelines for drinking-water quality. 4th ed. Geneva: WHO. Wilcox, L. V. 1955. Classification and use of irrigation water. Washington, DC: USDA.

Yamakanamardi, S. V., U. S. Hampannavar, and B. K. Purandara. 2011. "Assessment of chloride concentration in groundwater: A case study for Belgaum city." Int. J. Environ. Sci. 2 (1): 283-292. https://doi .org/10.6088/ijes.00202010028. 